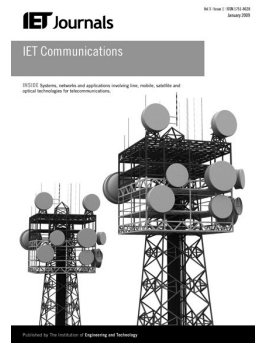

\title{
Performance analysis of high-speed railway is communication systems subjected to co-channel interference and channel estimation errors
}

\author{
Jiayi Zhang 1,2, Fan Jin'3, Zhenhui Tan 1,2, Haibo Wang1, Qing Huang1,4, Lajos Hanzo \\ $20{ }^{1}$ Institute of Broadband Wireless Mobile Communications, Beijing Jiaotong University, Beijing 100044, \\ People's Republic of China \\ ${ }^{2}$ State Key Laboratory of Rail Traffic Control and Safety, Beijing Jiaotong University, Beijing 100044, \\ People's Republic of China \\ ${ }^{3}$ School of Electrical and Computer Science, University of Southampton, Southampton SO17 1BJ, UK \\ $25{ }^{4}$ National Mobile Communications Research Laboratory, Southeast University, Nanjing 210096, \\ People's Republic of China \\ E-mail: Ih@ecs.soton.ac.uk
}

Abstract: The performance of high-speed railway wireless communication systems is studied in the presence of co-channel 30 interference and imperfect channel estimation in the uplink. The authors derive exact closed-form expressions for the outage probability and investigate the impact of fading severity. New explicit expressions are derived for both the level crossing rate and average outage duration for illustrating the impact of mobile speed and channel estimation errors on the achievable system performance. Our results are generalised and hence they subsume a range of previously reported results.

\section{Introduction}

40

Given the development of high-speed railways all over the globe, it becomes necessary to support public broadband wireless access to mobile terminals (MTs) aboard high-speed trains. Owing to the large penetration loss encountered, when providing coverage within the carriages from outdoor fixed station (FS) and owing to the huge handoff burden, it is a promising solution to install an on-board base station (BS) on the train to provide high-quality indoor coverage. The system architecture is depicted in Fig. 1, where the on-board BS relays the signals from the MTs to a trackside FS. We use the Nakagami- $m$ distribution as our small-scale fading model, which is typically considered as one of the most appropriate channel models. Moreover, a number of co-channel interferers (CCIs) surround the FS.

Recently, wireless communications for high-speed railway has attracted substantial interests [1-6]. Many challenges occur when designing this system. To elaborate a little further, the channel characteristics of high-speed railways have been studied in $[1,2]$. The authors argued that there is 60 always a line-of-sight signal path between the FS and the on-board BS in an open terrain scenario, which leads to Rician fading distribution. Nevertheless, the Nakagami- $m$ distribution includes both the Rician and Rayleigh distributions as special cases. Therefore we opted for using Nakagami- $m$ fading channel as our analytical model. The handoff problem, which is also challenging to the context of high-speed railway communications, has been analysed in $[3,4]$. With the drastic increase of train speeds, the handovers will occur more and more frequently, which potentially further degrades the achievable performance seriously. Furthermore, the energy efficiency and the fairness of high-speed railway communication systems have been considered in $[5,6]$. It is argued that the most efficient power allocation will assign all the power, when the train is nearest to the FS, which may impose a substantially great unfairness as a function of the time.

Diverse performance metrics have been used in wireless system design [7]. The outage probability (OP) is a first-order statistical characteristic defined as the probability that the received signal-to-interference-plus-noise ratio (SINR) is below a specified threshold value. In [8, 9], the OP was investigated in the presence of interferers for transmission over Nakagami- $m$ fading channels, which has also been applied in cognitive and relay-aided systems subjected to interference-limited Nakagami- $m$ fading channels [10-13]. Despite its benefits, the OP fails to provide holistic insights into the design of communication systems. As further practical measures, both the level crossing rate (LCR) and the average outage duration (AOD) were proposed in [14] for reflecting both the relative frequency and the duration of outages. The expressions derived for the LCR and AOD of maximal-ratio combining-aided systems subjected to CCI were presented in [15], but again in the absence of both channel estimation (CE) errors and noise. As a further advance, Hadzi-Velkov 


\section{System and channel model}

\subsection{System model}

From Fig. 1, the signals received by the trackside FS may be described as

$$
y=\sqrt{r_{0}} h_{0} s_{0}+\sum_{i=1}^{L} \sqrt{r_{i}} h_{i} s_{i}+N
$$

where $r_{0}$ and $r_{i}$ denote the average signal-to-noise ratio (SNR) of the desired signal $s_{0}$ and of the interfering signal $s_{i}$, respectively. Furthermore, $L$ and $N$ denote the number of interfering signals and the normalised thermal noise, respectively.

We assume that both the MS to FS link and the users imposing the interference on the FS, since they utilise the same frequency spectrum. The interference is subjected to Nakagami- $m$ fading models. The probability density function (PDF) of the instantaneous fading amplitude of $\alpha=|h|$, namely $p(\alpha)$, is given by [17]

$$
p(\alpha)=\frac{2 m^{m} \alpha^{2 m-1}}{\Omega^{m} \Gamma(m)} \exp \left(-\frac{m \alpha^{2}}{\Omega}\right)
$$

where $\Gamma(m)$ denotes the gamma function, whereas $m$ and $\Omega$ are the Nakagami- $m$ fading parameters. We assume furthermore that the transmission channel $h_{0}$ obeys the Nakagami- $m$ distribution of $h_{0} \sim \operatorname{Nakagami}\left(m_{0}, \quad \Omega_{0}\right)$, whereas the interfering channel $h_{i}$ obeys the Nakagami- $m$ distribution of $h_{i} \sim \operatorname{Nakagami}\left(m_{i}, \Omega_{i}\right)$. Let the average SNRs of the interfering signals are identical, that is, we have $r_{1}=r_{2}=\cdots=r_{L}=r_{I}$. Finally, note that the ratio of $m_{i}$ and $\Omega_{i}$ for all the interfering signals is the same, yielding $m_{1} / \Omega_{1}=m_{1} / \Omega_{1}, \ldots, m_{L} / \Omega_{L}$ [This is valid in the case where the interferers are approximately at the same distance from the receiver such as a single multi-antenna interferer or a cluster of co-located CCIs.].

\section{Q205}

\subsection{Imperfect channel estimation}

We consider imperfect $\mathrm{CE}$ at the receiver, where imperfect linear minimum mean-square error $\mathrm{CE}$ is performed. Here, we use the following model for the asymptotic estimated channel $\hat{h}_{0}$ at the receiver [18]

$$
h_{0}=\sqrt{1-\varepsilon^{2}} \hat{h}_{0}+\varepsilon h_{\mathrm{e}}
$$

where the CE error $h_{\mathrm{e}}$ is a complex Gaussian random variable independent of $h_{0}$, having a zero mean and a unit variance, while $\varepsilon \in[0,1]$ is a measure of the CE accuracy. Specifically, when we set $\varepsilon \neq 0$, there will be CE errors, hence we may rewrite (1) as

$$
y=\sqrt{r_{0}} \hat{h}_{0} s_{0}+\sum_{i=1}^{L} \sqrt{r_{i}} h_{i} s_{i}+N+\sqrt{r_{0}} h_{\mathrm{e}} s_{0}
$$

where the term $\left(N+\sqrt{r_{0}} h_{\mathrm{e}} s_{0}\right)$ may be viewed as the "virtual noise' at the receiver.

\section{Performance analysis}

In this section, the performance of the high-speed railway scenario of Fig. 1 is investigated in terms of its OP, LCR and $\mathrm{AOD}$, respectively.

\subsection{Outage probability}

We first determine the PDF of the instantaneous received SINR $\lambda$, then an exact OP expression is derived. The FS suffers from the interference imposed by the interfering macrocell users, which communicate in the same frequency band. As a result, the SINR $\lambda$ of the FS is given by

$$
\lambda=\frac{\left(1-\varepsilon^{2}\right) r_{0}\left|\hat{h}_{0}\right|^{2}}{r_{0} \varepsilon^{2}+1+\sum_{i=1}^{L} r_{I}\left|h_{i}\right|^{2}} \triangleq \frac{X}{C+Y}
$$

where $r_{0}$ and $r_{I}$ denote the average SNR of the desired signal $s_{0}$ and the interfering signal $s_{i}$, respectively. For the ease of analysis, we introduce the short-hand of $X \triangleq\left(1-\varepsilon^{2}\right) r_{0}\left|\hat{h}_{0}\right|^{2}, C \triangleq r_{0} \varepsilon^{2}+1$ and $Y \triangleq \sum_{i=1}^{L} r_{I}\left|h_{i}\right|^{2}$ as the power of the desired signal, the power of the virtual 255 noise and the power of the interfering signal, respectively.

Since the channel $\hat{h}_{0}$ obeys the Nakagami- $m$ distribution $\hat{h}_{0} \sim \operatorname{Nakagami}\left(m_{0}, \Omega_{0}\right)$, the power of the desired signal $X$ follows the Gamma distribution of $X \sim \operatorname{Gamma}\left(k_{1}, \theta_{1}\right)$, where we have $k_{1}=m_{0}$ and $\theta_{1}=\left(1-\varepsilon^{2}\right) r_{0}\left(\Omega_{0} / m_{0}\right)$. Similarly, the variable $Y$, which is the sum of $L$ independent Gamma distributed variables, also follows the Gamma distribution of $Y \sim \operatorname{Gamma}\left(k_{2}, \theta_{2}\right)$, where we have $k_{2}=\sum_{i=1}^{L} m_{i}$ and $\theta_{2}=\left(r_{I} \Omega_{i} / m_{i}\right)$. In (5), the PDF of $\gamma$ is 
written as

$$
f_{\lambda}(\lambda)=\int_{0}^{\infty}(y+C) f_{X}[(y+C) \lambda] f_{Y}(y) \mathrm{d} y
$$

270 where $f_{X}(x)=\left(1 / \theta_{1}^{k_{1}} \Gamma\left(k_{1}\right)\right) x^{k_{1}-1} \exp \left(-\left(x / \theta_{1}\right)\right)$ and $f_{Y}(y)=$ $\left(1 / \theta_{2}^{k_{2}} \Gamma\left(k_{2}\right)\right) x^{k_{2}-1} \exp \left(-\left(y / \theta_{2}\right)\right)$ denote the PDF of the variables $X$ and $Y$, respectively. We can derive the closed-form expression for the PDF of the received SINR $\lambda$, as detailed in Appendix 1. The OP $P_{\text {out }}$ can be obtained by integrating (6) with respect to $\lambda$ between the limits $0 \leq \lambda \leq$ $\lambda_{\text {th }}$, which is given by

$$
P_{\text {out }}\left(\lambda_{\text {th }}\right)=\int_{0}^{\lambda_{\text {th }}} \int_{0}^{\infty}(y+C) f_{X}[(y+C) \lambda] f_{Y}(y) \mathrm{d} y \mathrm{~d} \lambda
$$

According to Appendix 1, we may express the exact OP as

$$
\begin{aligned}
P_{\text {out }}\left(\lambda_{\text {th }}\right)= & 1-\frac{\exp \left(-\left(C \lambda_{\text {th }} / \theta_{1}\right)\right)}{\Gamma\left(k_{2}\right) \theta_{2}^{k_{2}}} \sum_{m=0}^{k_{1}-1}\left(\frac{\lambda_{\text {th }}}{\theta_{1}}\right)^{m} \frac{1}{m !} \\
& \times \sum_{n=0}^{m}\left(\begin{array}{c}
m \\
n
\end{array}\right) C^{m-n} \frac{\Gamma\left(n+k_{2}\right)}{\left(\left(\lambda_{\text {th }} / \theta_{1}\right)+\left(1 / \theta_{2}\right)\right)^{n+k_{2}}}
\end{aligned}
$$

Specifically, when we consider the perfect CE-aided interference-limited system, that is, $\varepsilon=0,(8)$ reduces to

295

$$
P_{\text {out }}\left(\lambda_{\text {th }}\right)=1-\frac{1}{\Gamma\left(k_{2}\right) \theta_{2}^{k_{2}}} \sum_{m=0}^{k_{1}-1}\left(\frac{\lambda_{\text {th }}}{\theta_{1}}\right)^{m} \frac{1}{m !} \frac{\Gamma\left(m+k_{2}\right)}{\left(\left(\lambda_{\text {th }} / \theta_{1}\right)+\left(1 / \theta_{2}\right)\right)^{m+k_{2}}}
$$

which is in agreement with [11, Eq. (25)], as expected.

\subsection{Level crossing rate}

Let us first define the ratio of the desired signal envelope $305 S \triangleq \sqrt{X}$ and the interference-plus-noise envelope $Z \triangleq \sqrt{C+Y}$ as

$$
g \triangleq \sqrt{\lambda} \triangleq \frac{S}{Z}
$$

310

The average LCR of the envelope ratio at a threshold of $g_{\text {th }}=\sqrt{\lambda_{\text {th }}}$ represents the average number of times the fading process $g$ crosses the threshold $g_{\text {th }}$ in the positive direction per unit time. The average LCR $N(g)$ can be obtained from the general formula provided in [19] as

$$
N(g)=\int_{0}^{\infty} \dot{g} f_{g, \dot{g}}(g, \dot{g}) \mathrm{d} \dot{g}
$$

where $\dot{g}$ denotes the time derivative of $g$ and $f_{g, \dot{g}}(g, \dot{g})$ is the joint PDF of the pair of variables $g$ and $\dot{g}$ in an arbitrary time slot $t$. To derive $f_{g, \dot{g}}(g, \dot{g})$, we choose the following transform

325

$$
\begin{gathered}
f_{g, \dot{g}}(g, \dot{g})=\int_{0}^{\infty} f_{g, \dot{g} \mid Z}(g, \dot{g} \mid z) f_{Z}(z) \mathrm{d} z \\
=\int_{0}^{\infty} f_{\dot{g} \mid g, Z}(\dot{g} \mid g, z) f_{g \mid Z}(g \mid z) f_{Z}(z) \mathrm{d} z
\end{gathered}
$$

$$
=\int_{0}^{\infty} f_{\dot{g} \mid g, Z}(\dot{g} \mid g, z) z f_{S}(g z) f_{Z}(z) \mathrm{d} z
$$

where $f_{g, \dot{g} \mid Z}(g, \dot{g} \mid z), \quad f_{\dot{g} \mid g, Z}(\dot{g} \mid g, z)$ and $f_{g / Z}(g \mid z)$ are the conditional joint PDF of the pair of variables $g$ and $\dot{g}$, given the specified value $Z=z$ and the conditional PDF of $\dot{g}$, given some specified value $g$ and $Z=z$, as well as the conditional PDF of $g$ given some specified value $Z$, respectively. Furthermore, $f_{S}(\cdot)$ and $f_{Z}(\cdot)$ are the PDFs of the variables $S$ and $Z$, respectively. Moreover, we proceed from (13) to (14) by exploiting (10). Then the time derivative of the envelope ratio may be written from (11) as

$$
\dot{g}=\frac{\dot{S}}{Z}-\frac{\dot{Z}}{Z} g
$$

We note that the variable $S$ follows the Nakagami- $m$ distribution. Furthermore, according to a Gaussian model [19], $\dot{S}$ obeys a zero-mean Gaussian distribution with a variance of $\sigma_{1}^{2}=\left(\pi f_{d_{0}}\right)^{2} \theta_{1}$, where $f_{d_{0}}$ denotes the maximum Doppler frequency shift of the desired signal. The maximum Doppler frequency shift $f_{d_{0}}$ can be expressed as $f_{d_{0}}=v f / c$, where $v$ is the speed of high-speed train, $f$ is the carrier frequency and $c$ is the speed of light in free space. Similarly, $Z^{2}=C+Y$ is a constant plus a squared Nakagami- $m$ random variable. Upon setting the derivatives of both sides with respect to $t$, the constant $C$ vanishes. As a result, the time derivative of the interference-plus-noise envelope $\dot{Z}$ also follows a zero-mean Gaussian distribution with a variance of $\sigma_{2}^{2}=\left(\pi f_{d_{I}}\right)^{2} \theta_{2}$, where $f_{d_{I}}$ denotes the maximum Doppler frequency shift of the interfering signal. Consequently, given a specific $g$ and $Z=z, \dot{g}$ of (15) is a zero-mean Gaussian random variable with a variance of

$$
\sigma_{\dot{g} \mid g, Z}^{2}=\frac{1}{z^{2}} \sigma_{1}^{2}+\frac{g^{2}}{z^{2}} \sigma_{2}^{2}
$$

Upon substituting (14) into (11), the average LCR may be rewritten as

$$
\begin{aligned}
N(g) & =\int_{0}^{\infty} z f_{S}(g z) f_{Z}(z) \mathrm{d} z \int_{0}^{\infty} \dot{g} f_{\dot{g} \mid g, Z}(\dot{g} \mid g, z) \mathrm{d} \dot{g} \\
& =\sqrt{\frac{\sigma_{1}^{2}+\sigma_{2}^{2} g^{2}}{2 \pi}} \int_{0}^{\infty} f_{S}(g z) f_{Z}(z) \mathrm{d} z
\end{aligned}
$$

Based on the derivation in Appendix 2, the closed-form expression of the average LCR is given by

$$
\begin{aligned}
N(g)= & 2 \sqrt{\frac{\sigma_{1}^{2}+\sigma_{2}^{2} g^{2}}{2 \pi}} \frac{g^{2 k_{1}-1} \exp \left(C / \theta_{2}\right)}{\Gamma\left(k_{1}\right) \Gamma\left(k_{2}\right) \theta_{1}^{k_{1}} \theta_{2}^{k_{2}}} \\
& \times \sum_{n=0}^{k_{2}-1}\left(\begin{array}{c}
k_{2}-1 \\
n
\end{array}\right)(-C)^{k_{2}-n-1}\left(\frac{g^{2}}{\theta_{1}}+\frac{1}{\theta_{2}}\right)^{-\left(k_{2}+n+0.5\right)} \\
& \times \Gamma\left[k_{2}+n+0.5, C\left(\frac{g^{2}}{\theta_{1}}+\frac{1}{\theta_{2}}\right)\right]
\end{aligned}
$$

where $\Gamma(\alpha, x)=\int_{x}^{\infty} \exp (-t) t^{\alpha-1} \mathrm{~d} t$ denotes the upper 
incomplete gamma function of [20, Eq. (8.350.2)]. When we consider an interference-limited system relying on perfect $\mathrm{CE}$ and contaminated only by $\mathrm{CCI}$, that is, $\varepsilon=0$, (18) reduces to [15, Eq. (13)].

\subsection{Average outage duration}

The average outage duration is defined as the average time that the receive SINR $\lambda$ remains below the pre-defined threshold $\lambda_{\text {th }}$, which may be expressed as

$$
T\left(\lambda_{\text {th }}\right)=P_{\mathrm{o}}\left(\lambda_{\mathrm{th}}\right) / N\left(\lambda_{\mathrm{th}}\right)
$$

With the aid of our closed-form OP expression (8) and the LCR formula (18), the explicit expression of AOD is readily derived.

\section{Numerical results}

In this section, we present numerical results for validating our analytical expressions given in Section 3. Specifically, we study the detailed impact of the fading parameters, mobility and $\mathrm{CE}$ errors on the performance of high-speed railway communication systems. For convenience, we assume that the interfering users are stationary and use the notation of $\rho \triangleq \sqrt{1-\varepsilon^{2}}$ for the correlation coefficient between the true channel coefficients and their estimates.

Fig. 2 examines the accuracy of the asymptotic estimated model (3) for Nakagami- $m$ fading channels under the assumption of Gaussian CE errors. As seen in Fig. 2, the approximate curves are hardly distinguished from the exact ones in a moderate range of $\rho$ and the accuracy is improved upon increasing the correlation coefficient. Hence, we conclude from Fig. 2 that the asymptotic CE model is applicable for our analysis presented in Section 2.

Fig. 3 illustrates the OP of high-speed railway communications in the presence of CE errors and CCI. The $\mathrm{OP}$ results for perfect $\mathrm{CE}$ based on (9) have also been plotted in Fig. 3. It is clear that the OP recorded for perfect $\mathrm{CE}$ constitutes the lower bound of the $\mathrm{CE}$ errors and the gap between them increases for larger values of $m_{0}$. Furthermore, we assume that the four interferers have an identical power, but they communicate over different Nakagami- $m$ fading channels. As the desired average SNR $r_{0}$ increases from 0 to $6 \mathrm{~dB}$, there is a significant reduction in the OP. The curves seen in Fig. 3 also show that the OP performance improves upon increasing the fading parameter $m_{0}$, which is expected, since the Nakagami- $m$ fading channels become more benign Gaussian channels in the limit, as we have $m_{0} \rightarrow \infty$.

The second-order statistics of the received signals of high-speed communication systems with and without $\mathrm{CE}$ errors are characterised in Figs. 4 and 5. Fig. 4 shows that there is a special SINR threshold $\overline{\lambda_{\text {th }}}$, where the maximum value of the average LCR is reached. For values of $\lambda_{\text {th }}$ below $\overline{\lambda_{\text {th }}}$, the average LCR increases as a function of the SINR threshold and $\rho$. Note that increasing the speed of trains will increase the LCR, hence the signal envelopes fluctuate more rapidly. For a given Doppler spread, the

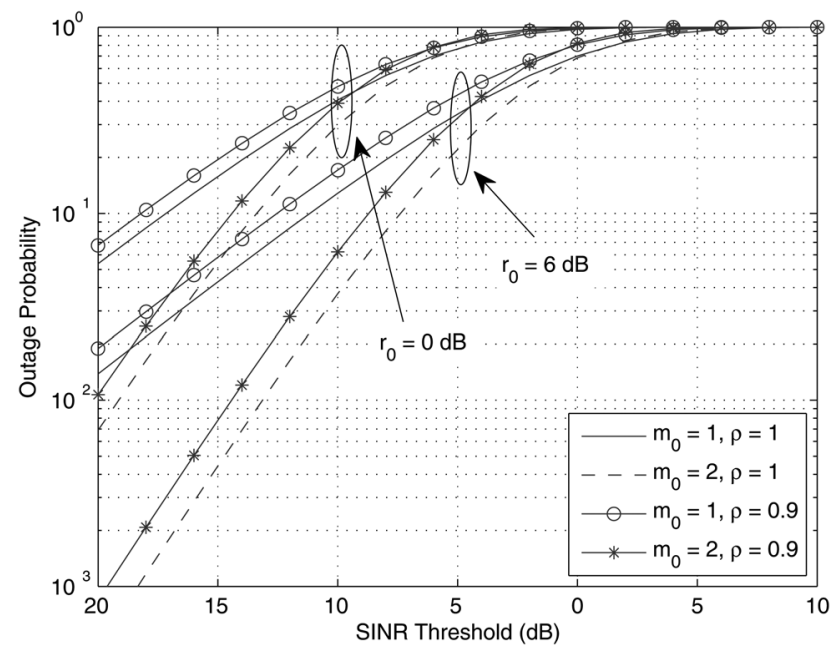

Fig. 3 OP against SINR threshold for different desired average SNR and fading parameters $\left(L=4, m_{i}=[0.5,1,0.5,1], \Omega_{i}=[1\right.$, $2,1,2]$ and $r_{I}=0 d B$ )
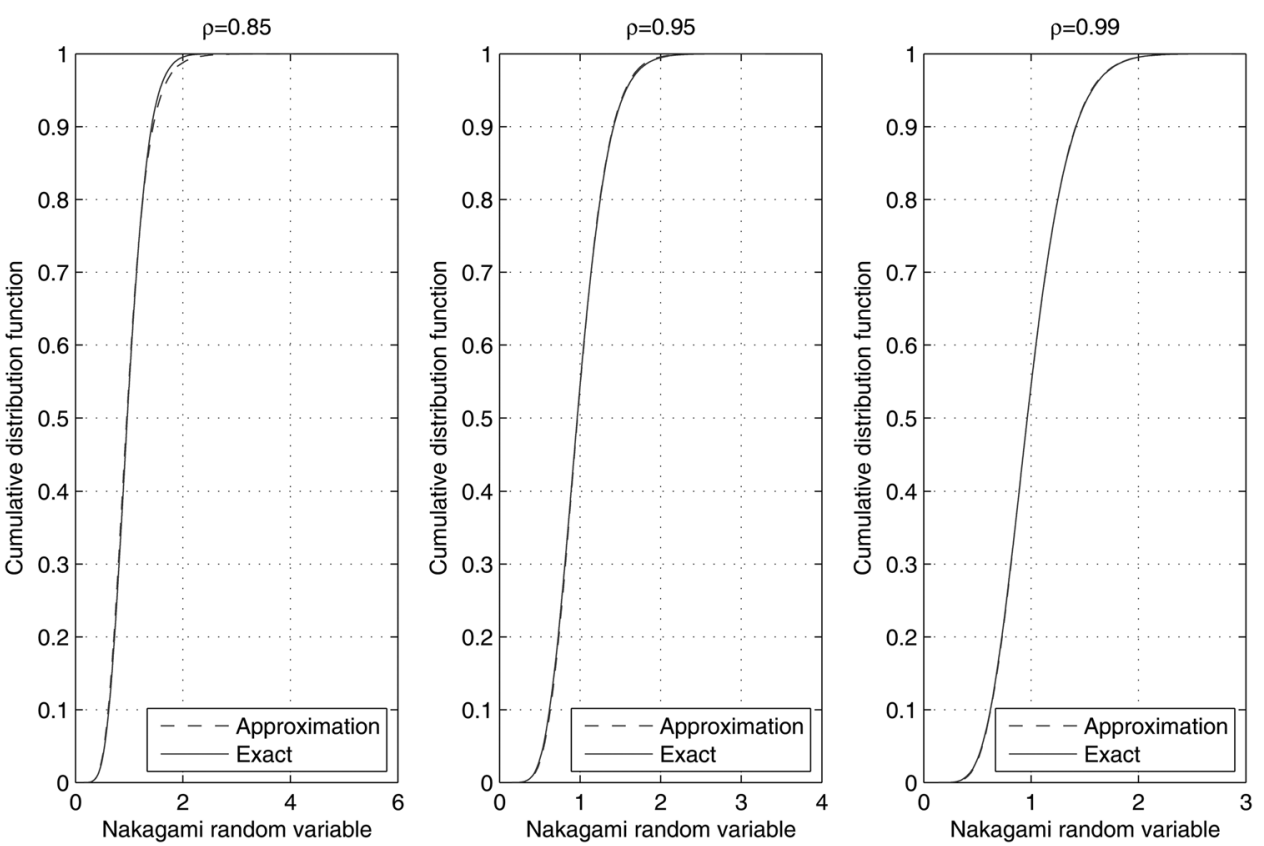

Fig. 2 Asymptotic CE model for different values of correlation coefficient $\rho$ 
Fig. 4 LCR against SINR threshold for different moving speed and CE errors $\left(L=4, m_{i}=[0.5,1,0.5,1], \Omega_{i}=[1,2,1,2], m_{0}=2, \Omega_{0}=\right.$ 1 and $\left.r_{I}=0 d B\right)$

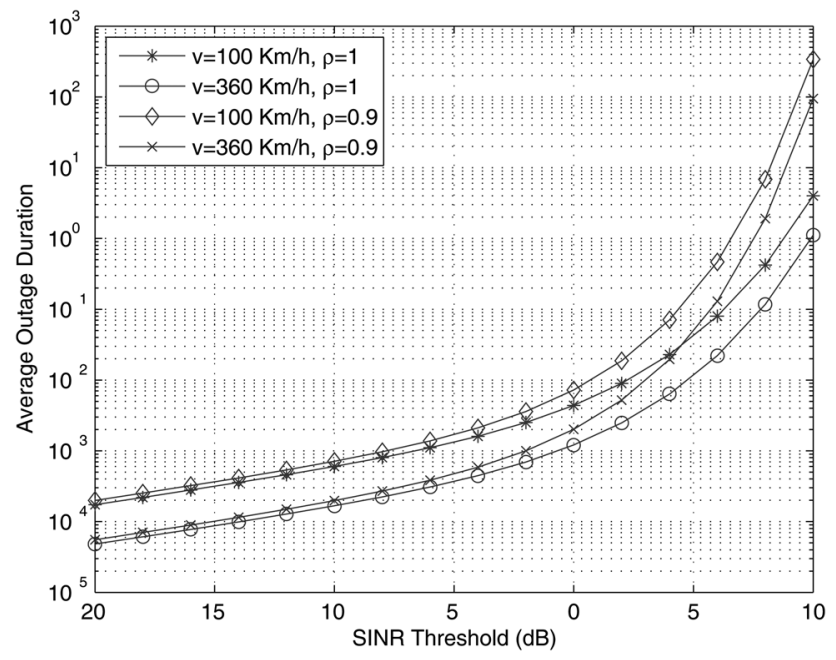

effect of CE errors are more grave in the high SINR threshold region. Furthermore, the gap between the LCRs found for perfect $\mathrm{CE}$ and for realistic $\mathrm{CE}$ errors becomes larger upon increasing the SINR threshold $\lambda_{\text {th }}$.

We can observe from Fig. 5 that the AOD is a monotonically increasing function of the SINR threshold. By increasing the CE accuracy, it can be seen that a modest improvement is experienced for $\lambda_{\text {th }}<\overline{\lambda_{\text {th }}}$, whereas a significant increase can be found for SINR thresholds higher than $\overline{\lambda_{\text {th }}}$. However, the speed of AOD improvement 585 recorded for perfect $\mathrm{CE}$ is slower than the one found for realistic CE errors. Fig. 5 also shows the effects of the speed of trains on the AOD. As expected, increasing the train's speed results in a reduction of the AOD.

\section{Conclusions}

In this paper, we investigated the first- and second-order statistical wave characteristics at the FS of high-speed railway communication systems in the presence of both the CCI and
CE errors. Both the signals received by the FS from the train and the interfering users are assumed to experience Nakagami- $m$ fading. The PDF of the SINR and the exact closed-form expression of the OP were derived in the form of finite sums. Moreover, we presented the exact closed-form expressions of both the LCR and AOD, which provided an efficient characterisation of the Doppler spread, the fading parameters and the interference and imperfect $\mathrm{CE}$ both on the LCR and on the AOD performance of this system. Naturally, severe fading conditions between the trains and the FS always degrades the OP performance, while may be compensated by increasing the power of the train's transmitter. In addition, the LCR experienced at high SINR thresholds increases as the train-speed is increased and the $\mathrm{CE}$ errors are reduced, which results in a reduced AOD.

\section{Acknowledgment}

This work has been supported in part by the National ST Major Project (2011ZX03005-004-03), the National Natural Science Foundation of China $(61071075,61001071)$ and the open research fund of National Mobile Communications Research Laboratory, Southeast University (No. 2013D03). The fiscal support of the European Research Council's Advanced Fellow grant is also gratefully acknowledged.

\section{References}

1 Liu, L., Tao, C., Qiu, J., et al.: 'Position-based modeling for wireless channel on high-speed railway under a viaduct at $2.35 \mathrm{GHz}$, IEEE J. Sel. Areas Commun., 2012, 30, (4), pp. 834-845

$2 \mathrm{He}, \mathrm{R}$. , Zhong, Z., Ai, B., et al.: 'Measurements and analysis of propagation channels in high-speed railway viaducts', IEEE Trans. Wirel. Commun., 2013, 12, (2), pp. 794-805

3 Wang, J., Zhu, H., Gomes, N.J.: 'Distributed antenna systems for mobile communications in high speed trains', IEEE J. Sel. Areas Commun., 2012, 30, (4), pp. 675-683

4 Tian, L., Li, J., Huang, Y., et al.: 'Seamless dual-link handover scheme in broadband wireless communication systems for high-speed rail', IEEE J. Sel. Areas Commun., 2012, 30, (4), pp. 708-718

5 Liang, H., Zhuang, W.: 'Efficient on-demand data service delivery to high-speed trains in cellular/infostation integrated networks', IEEE J. Sel. Areas Commun., 2012, 30, (4), pp. 780-791

6 Dong, Y., Fan, P., Letaief, K.: 'High speed railway wireless communications: efficiency vs. fairness', IEEE Trans. Veh. Technol., 2013. Available at http://ieeexplore.iee.org/stamp/stamp.jsp? $\mathrm{tp}=$ \&arnumber $=6595639$

7 Steele, R., Hanzo, L.: 'Mobile radio communications' (Pentech Press, London, 1999, 2nd edn.)

8 Zhang, Q.T.: 'Outage probability in cellular mobile radio due to Nakagami signal and interferers with arbitrary parameters', IEEE Trans. Veh. Technol., 1996, 45, (2), pp. 364-372

9 Aalo, V.A., Zhang, J.: 'Performance analysis of maximal ratio combining in the presence of multiple equal-power cochannel interferers in a Nakagami fading channel', IEEE Trans. Veh. Technol., 2001, 50, (2), pp. 497-503

10 Yang, Q., Kwak, K.S.: 'Outage performance of cooperative relaying with dissimilar Nakagami- $m$ interferers in Nakagami- $m$ fading', IET Commun., 2009, 3, (7), pp. 1179-1185

11 Zhong, C., Ratnarajah, T., Wong, K.K.: 'Outage analysis of decode-and-forward cognitive dual-hop systems with the interference constraint in Nakagami-m fading channels', IEEE Trans. Veh. Technol., 2011, 60, (6), pp. 2875-2879

12 Soithong, T., Aalo, V.A., Efthymoglou, G.P., et al.: 'Outage analysis of multihop relay systems in interference-limited Nakagami- $m$ fading channels', IEEE Trans. Veh. Technol., 2012, 61, (3), pp. 1451-1457

13 Fredj, K.B., Aissa, S., Musavian, L.: 'Ergodic and outage capacities of relaying channels in spectrum-sharing constrained systems', IET Commun., 2013, 7, (2), pp. 98-109

14 Stüber, G.L.: 'Principles of mobile communication' (Springer, New York, 2011, 2nd edn.)

15 Yang, L., Alouini, M.S.: 'On the average outage rate and average outage duration of wireless communication systems with multiple cochannel interferers', IEEE Trans. Wirel. Commun., 2013, 3, (4), pp. 1142-1153 
16 Hadzi-Velkov, Z.: 'Level crossing rate and average fade duration of dual selection combining with cochannel interference and Nakagami fading', IEEE Trans. Wirel. Commun., 2007, 6, (11), pp. 3870-3876

17 Nakagami, M.: 'The $m$-distribution: a general formula of intensity

Q4

distribution of rapid fading', Stat. Methods Radiowave Propag., 1960

18 Gifford, W.M., Win, M.Z., Chiani, M.: 'Diversity with practical channel estimation', IEEE Trans. Wirel. Commun., 2007, 4, (4), pp. 1935-1947

19 Rice, S.O.: 'Statistical properties of a sine wave plus random noise', Bell Syst. Tech. J., 2007, 27, pp. 109-157

20 Gradshteyn, I.S., Ryzhik, I.M.: 'Table of integrals, series, and products' (Academic Press, San Diego, 2007, 7th edn.)

\section{Appendix}

\subsection{Appendix 1: The derivation of the PDF and} cumulative distribution function of the received SINR

Upon substituting the PDF of the random variables $X$ and $Y$ into (6), the PDF of $\lambda$ may be expressed as

According to the Binomial theorem $(y+C)^{k_{1}}=\sum_{n=0}^{k_{1}}\left(\begin{array}{c}k_{1} \\ n\end{array}\right) y^{n} C^{k_{1}-n}$ [20, Eq. (1.111)], (20) may

$=\frac{\lambda^{k_{1}-1} \exp \left(-\left(C \lambda / \theta_{1}\right)\right)}{\Gamma\left(k_{1}\right) \Gamma\left(k_{2}\right) \theta_{1}^{k_{1}} \theta_{2}^{k_{2}}} \sum_{n=0}^{k_{1}}\left(\begin{array}{c}k_{1} \\ n\end{array}\right) C^{k_{1}-n} \frac{\Gamma\left(n+k_{2}\right)}{\left(\left(\lambda / \theta_{1}\right)+\left(1 / \theta_{2}\right)\right)^{n+k_{2}}}$

The cumulative distribution function (CDF) of the received SINR $\gamma$ may be expressed as

$$
F_{\lambda}(\lambda)=\int_{0}^{\lambda} f_{\lambda}(\lambda) \mathrm{d} \lambda
$$

However, this approach requires tedious mathematical manipulations. We may hence pursue a different approach to derive the exact expression, which may be shown to be

$$
\begin{aligned}
F_{\lambda}(\lambda)= & \int_{0}^{\lambda} \frac{\lambda^{k_{1}-1}}{\Gamma\left(k_{1}\right) \Gamma\left(k_{2}\right) \theta_{1}^{k_{1}} \theta_{2}^{k_{2}}} \int_{0}^{\infty}(y+C)^{k_{1}} y^{k_{2}-1} \\
& \times \exp \left[-\frac{(y+C) \lambda}{\theta_{1}}-\frac{y}{\theta_{2}}\right] \mathrm{d} y \mathrm{~d} \lambda \\
= & \frac{1}{\Gamma\left(k_{1}\right) \Gamma\left(k_{2}\right) \theta_{1}^{k_{1}} \theta_{2}^{k_{2}}} \int_{0}^{\infty} \int_{0}^{\lambda} \lambda^{k_{1}-1} \exp \left(-\frac{y+C}{\theta_{1}} \lambda\right) \\
& \times \mathrm{d} \lambda(y+C)^{k_{1}} y^{k_{2}-1} \exp \left(-\frac{y}{\theta_{2}}\right) \mathrm{d} y \mathrm{~d} \lambda
\end{aligned}
$$

Using the Binomial theorem, the closed-form expression for the $\mathrm{CDF}$ of $\lambda$ can be expressed as

$$
\begin{aligned}
F_{\lambda}(\lambda)= & 1-\frac{\exp \left(-\left(C \lambda / \theta_{1}\right)\right)}{\Gamma\left(k_{2}\right) \theta_{2}^{k_{2}}} \sum_{m=0}^{k_{1}-1}\left(\frac{\lambda}{\theta_{1}}\right)^{m} \frac{1}{m !} \\
& \times \sum_{n=0}^{m}\left(\begin{array}{c}
m \\
n
\end{array}\right) C^{m-n} \frac{\Gamma\left(n+k_{2}\right)}{\left(\left(\lambda_{\mathrm{th}} / \theta_{1}\right)+\left(1 / \theta_{2}\right)\right)^{n+k_{2}}}
\end{aligned}
$$

\subsection{Appendix 2: The derivation for the average $L C R$}

Let us now provide the derivation of the integral term in (17). Since the random variable $S$ follows the Nakagami- $m$ distribution, the PDF of $S$ is readily expressed as

$$
f_{S}(x)=\frac{2 x^{2 k_{1}-1}}{\Gamma\left(k_{1}\right) \theta_{1}^{k_{1}}} \exp \left(-\frac{x^{2}}{\theta_{1}}\right)
$$

Since we have $Z=\sqrt{Y+C}$, we may infer that $P[Z \leq z]=P$ $\left[Y \leq z^{2}-C\right]$. As mentioned in Section 3, $Y$ follows the Gamma distribution. As a result, the $\mathrm{CDF}$ of the variable $Z$ is given by

$$
f_{Z}(z)=\frac{2 z}{\Gamma\left(k_{2}\right) \theta_{2}^{k_{2}}}\left(z^{2}-C\right)^{k_{2}-1} \exp \left[-\frac{z^{2}-C}{\theta_{2}}\right]
$$

Note that the random variable $Y$ is always positive and $Z$ is ranging from $\sqrt{C}$ to $\infty$. By substituting (29) and (30) into 
(17), we have

795

$$
\begin{aligned}
N(g)= & \sqrt{\frac{\sigma_{1}^{2}+\sigma_{2}^{2} g^{2}}{2 \pi}} \int_{0}^{\infty} f_{S}(g z) f_{Z}(z) \mathrm{d} z \\
= & \sqrt{\frac{\sigma_{1}^{2}+\sigma_{2}^{2} g^{2}}{2 \pi}} \int_{\sqrt{C}}^{\infty} \frac{2(g z)^{2 k_{1}-1}}{\Gamma\left(k_{1}\right) \theta_{1}^{k_{1}}} \exp \left[-\frac{(g z)^{2}}{\theta_{1}}\right] \\
& \times \frac{2 z}{\Gamma\left(k_{2}\right) \theta_{2}^{k_{2}}}\left(z^{2}-C\right)^{k_{2}-1} \exp \left[-\frac{z^{2}-C}{\theta_{2}}\right] \mathrm{d} z
\end{aligned}
$$

805

$$
\begin{aligned}
= & 2 \sqrt{\frac{\sigma_{1}^{2}+\sigma_{2}^{2} g^{2}}{2 \pi}} \frac{g^{2 k_{1}-1} \exp \left(C / \theta_{2}\right)}{\Gamma\left(k_{1}\right) \Gamma\left(k_{2}\right) \theta_{1}^{k_{1}} \theta_{2}^{k_{2}}} \\
& \times \int_{C}^{\infty} z^{k_{1}-0.5}(z-1)^{k_{1}-1} \exp \left[-\left(\frac{g^{2}}{\theta_{1}}+\frac{1}{\theta_{2}}\right) z\right] \mathrm{d} z
\end{aligned}
$$

Using the Binomial theorem and [20, Eq. (3.351.2)], the explicit solution of (31) is given by (18). 


\section{www.ietdl.org}

${ }^{925}$ COM20130674

\section{Author Queries}

Jiayi Zhang, Fan Jin, Zhenhui Tan, Haibo Wang, Qing Huang, Lajos Hanzo

930 Q1 Please check and confirm the changes made to the sentence 'It is argued that...'

Q2 All footnotes have been moved to text as required by journal style. Please check and confirm that they have been located correctly within the text.

Q3 Please provide volume number and page range for reference [6].

Q4 Please provide volume and page range for Ref. [17]. 